\title{
Some properties of the twisted Grassmann graphs
}

go back

full screen

close

quit

Tatsuya Fujisaki* Jack H. Koolen Makoto Tagami

\begin{abstract}
In this note we determine the full automorphism group of the twisted Grassmann graph. Further we show that twisted Grassmann graphs do not have antipodal distance-regular covers. At last, we show that the twisted Grassmann graphs are not the halved graphs of bipartite distance-regular graphs.
\end{abstract}

Keywords : automorphism group twisted Grassmann graph, antipodal covers, bipartite MSC 2000: 05E20, 05E30, 05C25

\section{Introduction}

In November 2004, E. van Dam and J. Koolen [4] constructed the twisted Grassmann Graphs. These graphs have the same parameters as the Grassmann graphs $J_{q}(2 e+1, e)$, but are not vertex-transitive. In this note we will determine the full automorphism groups of these graphs, and also show that they do not have distance-regular antipodal covers. Furthermore we show that they are not the halved graphs of bipartite distance-regular graphs.

In Section 2 we will give the preliminaries and definitions, in Section 3 we recall the twisted Grassmann graphs and their maximal cliques, in Section 4 we determine the automorphism group and in Section 5 and 6, respectively we show that they do not have distance-regular antipodal covers and are not the halved graphs of bipartite distance-regular graphs.

\footnotetext{
*This work was done while the first author visited the Combinatorial and Computational Mathematics Center at POSTECH. The first two authors were supported by the SRC/ERC program of MOST/KOSEF (R11-1999-054)
} 


\section{Definitions and preliminaries}

We begin this section by recalling some facts concerning distance-regular graphs (for more details see [1]). Suppose that $\Gamma$ is a connected graph. The distance $d(u, v)$ between any two vertices $u, v$ in the vertex set $V \Gamma$ of $\Gamma$ is the length of a shortest path between $u$ and $v$ in $\Gamma$. For any $v \in V \Gamma$, define $\Gamma_{i}(v)$ to be the set of vertices in $\Gamma$ at distance precisely $i$ from $v$, where $i$ is any non-negative integer not exceeding the diameter $D$ of $\Gamma$. In addition, define $\Gamma_{-1}(v)=\Gamma_{D+1}(v):=\emptyset$.

Following [1], we call $\Gamma$ distance-regular if there are integers $b_{i}, c_{i}, 0 \leq i \leq D$, such that for any two vertices $u, v \in V \Gamma$ at distance $i=d(u, v)$, there are precisely $c_{i}$ neighbors of $v$ in $\Gamma_{i-1}(u)$ and $b_{i}$ neighbors of $v$ in $\Gamma_{i+1}(u)$. In particular, $\Gamma$ is regular with valency $k:=b_{0}$. The numbers $c_{i}, b_{i}$ and

$$
a_{i}:=k-b_{i}-c_{i} \quad(i=0, \ldots, D),
$$

the number of neighbors of $v$ in $\Gamma_{i}(u)$ for $d(u, v)=i$, are called the intersection numbers of $\Gamma$.

Let $\Gamma$ be a distance-regular graph, with diameter $D$ and $n$ vertices. A partition $\Pi=P_{1}, P_{2}, \ldots, P_{f}$ of the vertex set $V \Gamma$ is called equitable if there are constants $\alpha_{i j}(i, j \in\{1, \ldots, f\})$ such that for any $x \in P_{i}$ the number of neighbours of $x$ in $P_{j}$ equals $\alpha_{i j}$.

A code $C$ in $\Gamma$ is just a subset of $V \Gamma$. For a vertex $x$ and a code $C$ define $d(x, C)=\min \{d(x, y) \mid y \in C\}$. For $i \leq D$ define $C_{i}=\{x \in V \Gamma \mid d(x, C)=i\}$. The covering radius of $C, \rho$ is defined as

$$
\rho=\max \left\{i \mid C_{i} \neq \emptyset\right\}
$$

A code $C$ is called completely regular if $\left\{C_{i} \mid 0 \leq i \leq \rho\right\}$ is an equitable partition of $\Gamma$.

Let $\Gamma$ be a distance-regular graph. Let $M_{V \Gamma}(\mathbb{C})$ be the matrix algebra indexed by $V \Gamma$ over $\mathbb{C}$. The matrix $A_{i}$ denotes the $i$-th adjacency matrix of $\Gamma$, that is to say that, $A_{i}$ is the matrix in $M_{V \Gamma}(\mathbb{C})$ whose $(x, y)$-entry is 1 if $d(x, y)=i$, and 0 otherwise.

Let $\mathfrak{A}$ be the subalgebra of $M_{V \Gamma}(\mathbb{C})$ generated by the adjacency matrix $A_{1}$. Then, for all $i$, the matrix $A_{i} \in \mathfrak{A}$. Since $A_{0}, \ldots, A_{D}$ are pairwise commutative normal matrices, they can be diagonalized simultaneously. It is well-known that the number of the maximal common eigenspaces of $A_{0}, \ldots, A_{D}$ is $D+1$ and $\operatorname{span}(1,1, \ldots, 1)$ is one of the maximal common eigenspaces. Denote the maximal common eigenspaces by $V_{0}=\langle(1,1, \ldots, 1)\rangle, V_{1}, \ldots, V_{D}$ and let $E_{i}$ be the orthogonal projection $\mathbb{C}^{|V \Gamma|} \rightarrow V_{i}$ expressed in the matrix form with respect to the unit vectors. Then $E_{0}, \ldots, E_{D}$ are the primitive idempotents of $\mathfrak{A}$. Let 



